\title{
CONTROLLER DESIGN TECHNIQUES FOR THE LOTKA-VOLTERRA NONLINEAR SYSTEM
}

\author{
Magno Enrique Mendoza Meza* \\ magno@pee.coppe.ufrj.br
}

\author{
Amit Bhaya* \\ amitenacad.ufrj.br
}

\author{
Eugenius Kaszkurewicz* \\ eugenius@nacad.ufrj.br
}

*Dept. of Electrical Engineering, COPPE,

Federal University of Rio de Janeiro,

P.O. Box 68504, RJ 21945-970, BRAZIL

\begin{abstract}
A large class of predator-prey models can be written as a nonlinear dynamical system in one or two variables (species). In many contexts, it is necessary to introduce a control into these dynamics. In this paper we focus on models of two species, and assume, as is common in mathematical ecology, that the control corresponds to a proportional removal of the predator population. Six controller design techniques are applied to the Lotka-Volterra model, which is thus used as a benchmark to evaluate and compare these techniques in an ecological context.
\end{abstract}

KEYWORDS: Adaptive control of oscillations, Control Liapunov function, Immersion and Invariance, Induced internal feedback, Static sliding-mode control, Uncertain inputs.

\section{RESUMO}

Uma ampla classe de modelos do tipo predador-presa pode ser escrita como um sistema dinâmico não linear em uma ou duas variáveis (espécies). Em diversos contextos é necessário introduzir um controle nessas dinâmicas. Este artigo foca-se em modelos de duas variáveis. Assume-se, de acordo com a praxe em ecologia matemática, que o controle corresponde à remoção de uma proporção da população dos predadores (controle proporcional). Seis técnicas de projeto de controladores são aplicados ao modelo Lotka-Volterra, o qual é utilizado como um padrão ou "benchmark" para ava- liar e comparar estas técnicas em um contexto ecológico.

PALAVRAS-CHAVE: Controle adaptativo de oscilações, Controle a modo deslizante estático, Entradas incertas, Função de Liapunov com controle, Imersão e invariância, Realimentação interna induzida.

\section{INTRODUCTION}

Physical, chemical and biological systems are inherently nonlinear (May, 1973; Slotine e Li, 1991; Khalil, 1992; Utkin, 1992). A large class of models that describe predatorprey population dynamics can be described as a nonlinear dynamic system. In this paper models of two species are considered and they have the following generic form

$$
\begin{aligned}
\dot{x} & =f_{1}(x)+f_{2}(x) y \\
\dot{y} & =f_{3}(x) y
\end{aligned}
$$

where the state variable $x$ denotes the prey density, the state variable $y$ denotes the predator density, the functions $f_{1}$ and $f_{3}$ describe the growth functions of the prey and predator respectively and $f_{2}$ is a predator consumption function.

One of the simplest models of predator-prey interaction was formulated in the 1920s by A. J. Lotka and V. Volterra and is thus known as the Lotka-Volterra model. It has been extensively studied because it is a paradigm for more realistic 
models, and has the following form

$$
\left\{\begin{array}{l}
\dot{x}=r_{1} x-a x y, \\
\dot{y}=-r_{2} y+b x y,
\end{array}\right.
$$

where the parameter $r_{1}$ is the growth rate of the prey, $r_{2}$ is the mortality rate of the predator, $a, b$ represent the interaction coefficients between the species; all parameters are positives, $f_{1}=r_{1} x, f_{2}=-a x, f_{3}=-r_{2}+b x$. These equations constitute the simplest representation of the essence of the nonlinear predator-prey interaction (May, 1973; Gurney e Nisbet, 1998).

There have been many attempts to consider changing the Lotka-Volterra dynamics (3) by the introduction of controls and the main objective of this paper is to briefly present both techniques that have already been used, as well as some that have not and compare them with a new technique proposed in this paper.

We will briefly discuss the other techniques in section 3 . Here we limit ourselves to a brief description of the proposed control.

\section{Population dynamic models with threshold control}

The paper focuses on the introduction of an exogenous control in models of populational dynamics of two species. The general model is as follows:

$$
\begin{aligned}
\dot{x} & =f_{1}(x)+f_{2}(x) y, \\
\dot{y} & =f_{3}(x) y-y u_{2},
\end{aligned}
$$

where the control $u_{2}$ corresponds to a proportional removal of the predator population. We note that the dynamical system (4), (5) is in the so called regular form (Utkin, 1992), also called triangular or chained form. We can choose $y=\hat{y}$ to control the subsystem (4) so that $x$ has some desired behavior, and then design $u_{2}$ so that $y$ in (5) tracks $\hat{y}$ which is the desired "input" for (4). However, in an ecological context, the controlling action $u_{2}$ must satisfy certain restrictions or desirable characteristics that are discussed in the following section.

\section{DESIRABLE CHARACTERISTICS OF CONTROL IN AN ECOLOGICAL CON- TEXT}

Throughout this paper, the control term is to be understood as removal of a certain species.

In this context, the control must have the following characteristics:
- Implementation simplicity: (i) the mathematical expression of the control must be as simple as possible, (ii) the control must not depend on the system parameters so that they do not need to be estimated.

- Nonnegative control. This corresponds to the proportional removal of one of the species. In other words, it is assumed that the control corresponds only to removal, i.e. we consider "harvesting" of a certain species.

- Minimal monitoring. Refers to the number of population densities that need to be monitored to implement a certain control. In the context of the two species model (4), (5) if only one density is used to design feedback, we refer to this as output feedback; if both densities are used, then we call this state feedback.

- Promotion of coexistence. Both species must reach sustainable equilibrium levels, in which the populations, in appropriate units, are both positive.

Finally, as far as units are concerned, note the following:

Density unit: The population density is the size of the population in relation to some space unit. Generally it is evaluated as the number of individuals or a population biomass, per unit area or volume.

Time unit: Time in ecological systems is usually measured in days, weeks or years.

\section{GENERAL APPROACHES FOR CON- TROL OF NONLINEAR DYNAMIC SYS- TEMS}

In this section we briefly present six different techniques of nonlinear system design applied to the Lotka-Volterra (3) as a benchmark, with the objective of comparing them to the proposed control.

The set of general design methods of controllers for nonlinear systems can be divided in two subsets in the ecological context, as follows:

\section{Techniques already applied to population dynam-} ics:

- Several papers on the control of nonlinear ecological system models under perturbations have been devoted to the study of vulnerability and non-vulnerability of ecosystems subjected to continual, unpredictable, but bounded disturbances due to changes in climatic conditions, diseases, migrating species, etc. (Beddington e May, 1977; Lee e Leitmann, 1983; Steele e Henderson, 1984; Vincent et al., 1985). 
- Fradkov e Pogromsky (1998) applied the so called speed gradient method of adaptive control of oscillations to control the populations of two competitive species. Their method was specific to the LotkaVolterra model of population dynamics.

- Emel'yanov et al. (1998) presented a general methodology, referred to as induced internal feedback, for the control of uncertain nonlinear dynamic systems. It is based on on-off control as well as continuous versions of the latter and applied to the Lotka-Volterra system.

- The proposed control is based on the application of control Liapunov functions (Sontag, 1989), exploring the structure of the predator-prey systems and the backstepping idea (Sepulchre et al., 1997) for the regular form (Utkin, 1992), as well as using the concept of real and virtual equilibria (Costa et al., 2000) to derive an on-off or variable structure control.

\section{Techniques not previously applied to population dynamics:}

- Junger e Steil (2003) presented a new type of sliding motion which results from a special choice of the sliding surfaces. They define sliding surfaces such that these become explicitly dependent on the outputs of the discontinuous block. Under this design, a special sliding mode characterizes the system dynamics, which they named static sliding mode, because it occurs along the static contour of the closed-loop systems.

- A new method to design asymptotically stabilizing and adaptive control laws for nonlinear systems is presented in Astolfi e Ortega (2003). The method relies upon the notions of system immersion and manifold invariance and, in principle, does not require the knowledge of a (control) Lyapunov function.

\subsection{Design of the controller according to Emel'yanov et al.}

The following theorem from Emel'yanov et al. (1998) is presented.

Theorem 1 Consider the system

$$
\begin{aligned}
& \dot{x}=\varphi_{1}(x, y) \\
& \dot{y}=\varphi_{2}(x, y)+B(t) u
\end{aligned}
$$

$z=\left[\begin{array}{ll}x & y\end{array}\right]^{T}, B=\operatorname{diag}\left(b_{1}, \ldots, b_{n}\right), b_{i}(\cdot) \in\left[\frac{1}{L}, L\right], \varphi_{1}, \varphi_{2}$ are continuous, Lipschitz locally and unknown.

There is a continuous control $\mathbf{u}$ such that the trajectories of the system (6) approach the set $G$ and enter in it in finite time, where

$$
G:=\{z:\|s\| \leq \sigma(x, t)\}
$$

with

$$
s:=y-v(x, t),
$$

in closed loop, $v(x, t)$ is the trajectory to be tracked, $\sigma(x, t)$ is a tracking error tolerance. The control has the following form:

$$
u=-\frac{s}{\|s\|} \Psi(\|s\|, \sigma) F(z, t) .
$$

\section{Emel'yanov et al. procedure applied to the Lotka-Volterra model with control only in the predator}

Consider system (3) with control applied only to the predator:

$$
\left\{\begin{array}{l}
\dot{x}=r_{1} x-a x y \\
\dot{y}=-r_{2} y+b x y-u .
\end{array}\right.
$$

Procedure:

1. Choose the equilibrium point, at which is desired to stabilize the system, for a prey density $M$. It must satisfy

$$
M>\sup \left(\frac{r_{2}}{b}\right),
$$

and the predator density must satisfy

$$
y^{e q}=\frac{r_{1}}{a} .
$$

2. Suppose that it is necessary to maintain $x$ close to $M$, i.e., $|x-M|<\delta$. Introduce the constants $L_{1}, L_{2}$ and $\sigma$ ( $\sigma$ as an induced error tolerance, in this case a constant). The constants must satisfy

$$
L_{1}+\sigma<\frac{r_{1}}{a}, \quad L_{2}-\sigma>\frac{r_{1}}{a} .
$$

3. Choose the internal feedback operator $v(x)$, e.g. see Figure 1,

$$
v(x)=L_{1}+\left(L_{2}-L_{1}\right) \max \left\{0, \min \left\{1, \frac{x-M+\delta}{2 \delta}\right\}\right\} .
$$

4. The "induced error vector" is defined as:

$$
s:=y-v(x) .
$$

5. The induced error tolerance $\sigma(x, t)$ is chosen such that:

$$
\|s\| \leq \sigma(x, t) .
$$




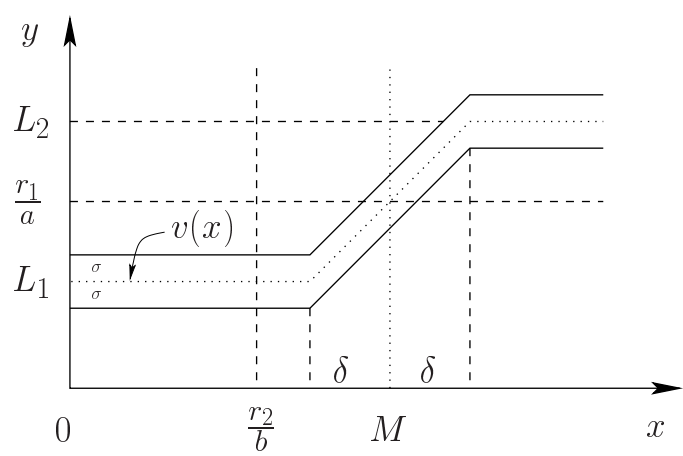

Figure 1: Induced internal feedback operator.

6. Check the conditions

$$
\left|\frac{d y}{d x}\right|>\left|\frac{d v}{d x}\right|
$$

to guarantee the invariance of region $G:=\{z:\|s\| \leq$ $\sigma\}$.

7. Analyze the behavior of the system in the following regions:

$$
|x-M|>\delta, \quad|x-M|<\delta .
$$

From the analysis, we obtain the gain $F$.

8. The control law is:

$$
u=F x y \max \left\{0, \min \left\{1, \frac{s-\sigma}{2 \sigma}\right\}\right\} .
$$

In this case the following restriction (see Emel'yanov et al. (1998) for details) must be satisfied:

$$
x^{e q}-\sigma>\sup \frac{r_{2}}{b} \text {. }
$$

For comparison, we use the parameters in Costa et al. (2000), substituting the values of these parameters in the constraints, the following numerical relations are obtained:

$$
x^{e q}>1.2, \quad F>b+\frac{L_{2}-L_{1}}{2 \delta} a=1.625 .
$$

Under these constraints, feasible values of desired equilibrium point as well as of the control effort are chosen

$$
\begin{aligned}
x^{e q} & =1.25, \quad y^{e q}=1, \quad L_{1}=0.75, \quad L_{2}=1.25, \\
F & =1.625, \quad M=1.25, \delta=\sigma=0.2 .
\end{aligned}
$$

The chosen value of $x^{e q}$ is the same used in Costa et al. (2000). The control is given by:

$$
u=F x y \max \left\{0, \min \left\{1, \frac{s-\sigma}{2 \sigma}\right\}\right\}
$$

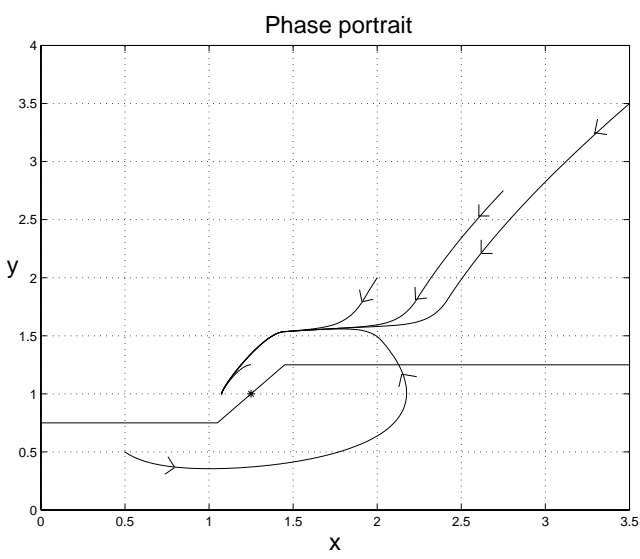

(a)

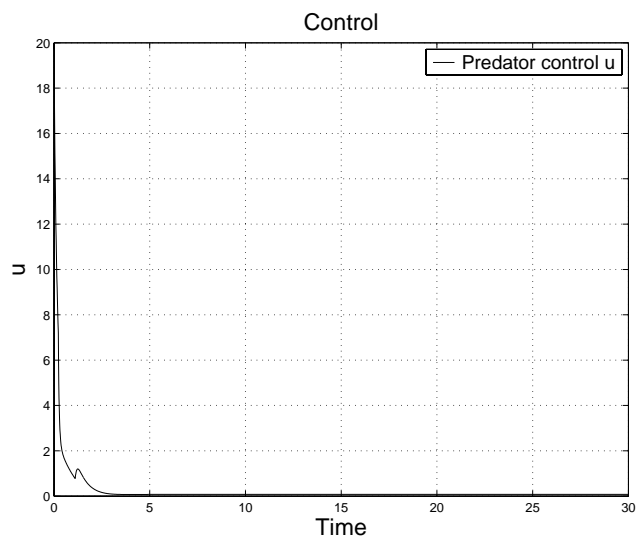

(b)

Figure 2: (a) Phase plane of Lotka-Volterra model subject to Emel'yanov's control. (b) Time evolution of the control action $u$. Parameter values $r_{1}=1, r_{2}=1, a=1, b=1, F=1.625, \delta=0.2$ and $\sigma=0.2$.

\subsection{Proposed Control design}

The idea of backstepping will be explained in a simple form for equations (7), (8). The state variable $y$ is taken as a fictitious input (fictitious control), denoted as $u_{1}$, to the prey subsystem (7). A control Liapunov function (CLF) is used to design the control $u_{1}$ such that the prey subsystem stabilizes in the desired equilibrium (for the prey). The next step is to design the (real control) $u_{2}$, involving removal of predators, such that the state of predator subsystem $y$ tracks the design input $u_{1}$. Again, the design is made using another CLF. In accordance with the observation that the control has to be maintained as simple as possible, both CLF's are chosen as quadratic functions. 
The resulting control system is described by:

$$
\begin{aligned}
\dot{x} & =f_{1}(x)+f_{2}(x) y \\
\dot{y} & =f_{3}(x) y-y u_{2}
\end{aligned}
$$

in which $u_{2}$ is the control (=threshold policy) to be designed. In other words, choose:

$$
u_{2}=\varepsilon_{2} \phi(\tau, \sigma),
$$

with $\varepsilon_{2}$ a control effort parameter to be designed and $\phi(\tau, \sigma)$ defined as,

$$
\phi(\tau, \sigma)=\left\{\begin{array}{lll}
1 & \text { if } & \tau>0 \\
0 & \text { if } & \tau<0,
\end{array}\right.
$$

where $\tau$ is a variable that defines the threshold, which is dependent on the system states.

The design of the CLF proceeds as follows: In the first subsystem (7), let $y=u_{1}$ be a fictitious control. Choose a CLF as

$$
V_{1}(x)=\frac{1}{2}\left(x-x_{d}\right)^{2}
$$

where $x_{d}$ is the desired equilibrium for the first subsystem. Note that a coordinate change can be made such that the desired value $x_{d}$ occurs at the origin.

Calculating the derivative of $V_{1}$ along the trajectories of (7) gives:

$$
\dot{V}_{1}=\left(x-x_{d}\right)\left(f_{1}(x)+f_{2}(x) u_{1}\right) .
$$

Now, assume for simplicity that $u_{1}$ is proportional to the prey density $x$, i.e.,

$$
u_{1}=\varepsilon x .
$$

Then the parameter $\varepsilon$ must be chosen such that $\dot{V}_{1}<0$.

Now, $u_{2}$ must be chosen such that $u_{1}$ satisfies (13). Therefore, choose the threshold $\tau$ as follows

$$
\tau=y-u_{1}=y-\varepsilon x,
$$

and a CLF $V_{2}$ as

$$
V_{2}=\frac{1}{2} \tau^{2}
$$

with the objective of maintaining $\tau=0$ and thus satisfying (13).

The derivative $V_{2}$ along the trajectories of (7), (8) leads to

$$
\dot{V}_{2}=\tau\left[\begin{array}{ll}
-\varepsilon & 1
\end{array}\right]\left[\begin{array}{c}
f_{1}(x)+f_{2}(x) y \\
f_{3}(x) y-y u_{2}
\end{array}\right] .
$$

Now the specific properties of functions $f_{1}, f_{2}$ and $f_{3}$ are used to choose $\varepsilon$ and $\varepsilon_{2}$ such that both functions satisfy $\dot{V}_{1}<$ 0 and $\dot{V}_{2}<0$, proving stability. The details of the choice and stability proofs can be found in Meza (2004) and have not been included in this paper for lack of space.

\section{Simulation of the behavior of the Lotka- Volterra model subject to the horizontal threshold policy applied only to the preda- tor}

The Lotka-Volterra subject to the threshold policy applied only to the predator stabilizes the system around the threshold as shown in the phase plane in Figure 3.a. Time evolution of the control is shown in Figure 3.b. The sliding equilibrium is $\left(x_{s l}^{e q}, y_{s l}^{e q}\right)=(1.25,1)$. In this case $\tau$ is chosen as $\tau=y-y_{s l}^{e q}$.

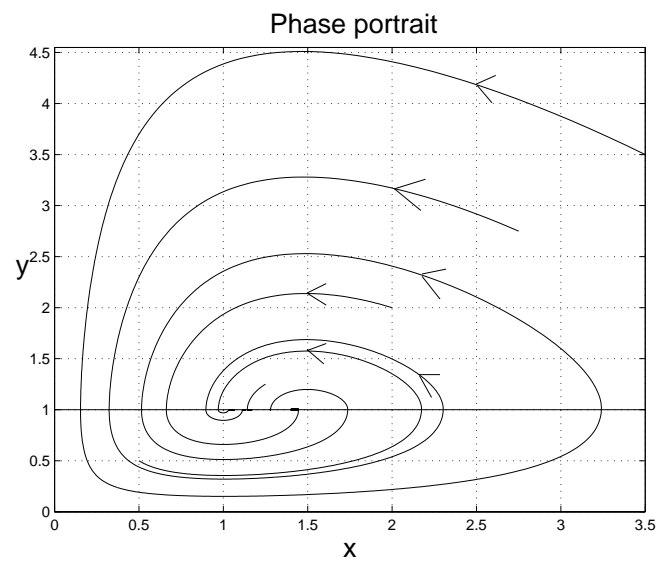

(a)

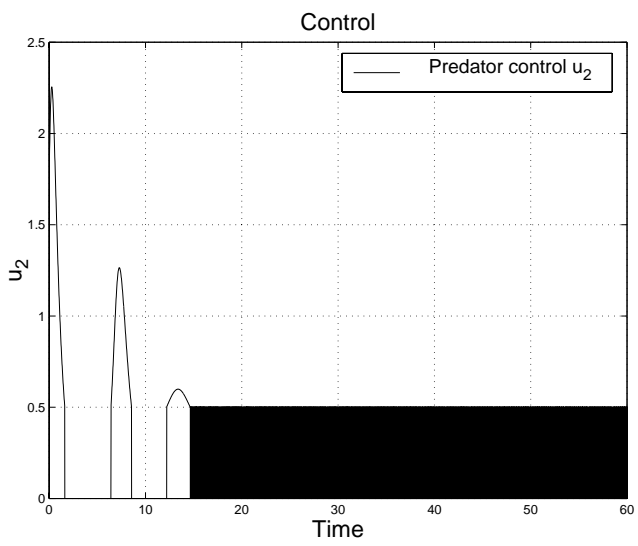

(b)

Figure 3: (a) Phase plane of the Lotka-Volterra model subject to the threshold policy. (b) Time evolution of the controlling action. Parameter values $r_{1}=1, r_{2}=1, a=1, b=1$ e $\varepsilon_{2}=0.5$

\subsection{Design of the controller according to Fradkov et al.}

Consider the Lotka-Volterra model as in (3), in which it is supposed that the birth rate of predator can be controlled. Fradkov e Pogromsky (1998) designed the control of the 
birth rate of the prey. In this case model (3) is modified as follows:

$$
\left\{\begin{array}{l}
\dot{x}=r_{1} x-a x y \\
\dot{y}=-r_{2} y+b x y+y u
\end{array}\right.
$$

where $u$ is the controlling action.

It is not difficult to show that the uncontrolled system $(u \equiv 0)$ has an infinite number of periodic solutions, provided that $x(0)>0, y(0)>0$, which correspond to the existence of the following first integral:

$$
\begin{aligned}
W(x, y)= & \left(b x-r_{2}-r_{2} \log \left(\frac{b x}{r_{2}}\right)\right) \\
& +\left(a y-r_{1}-r_{1} \log \left(\frac{a y}{r_{1}}\right)\right) .
\end{aligned}
$$

Indeed,

$$
\dot{W}(x, y)=0
$$

along any solution of $(3)(x(0)>0, y(0)>0)$, which means that the quantity $W$ preserves its constant value. The first integral (18) can be interpreted as a "total energy" of the "predator-prey" system and the control goal can be stated in terms of achieving the desired level of quantity $W$

$$
W(x(t), y(t)) \rightarrow W_{*} \quad \text { as } \quad t \rightarrow \infty .
$$

where $W_{*}$ is the desired level of the first intergral.

A control goal of this kind can be achieved by the speed gradient (SG) method, see Fradkov e Pogromsky (1998, Chap. 2). Introduce the following objective function $Q$ : $\mathbb{R} \times \mathbb{R} \rightarrow \mathbb{R}_{+}$:

$$
Q(x, y)=\frac{1}{2}\left(W(x, y)-W_{*}\right)^{2} .
$$

Then its time derivative with respect to the system (17) gives

$$
\dot{Q}(x, y)=\left(W(x, y)-W_{*}\right)\left(a y u-r_{2} u\right) .
$$

Calculating the gradient in $u$ gives:

$$
\frac{\partial \dot{Q}}{\partial u}(x, y)=\left(W(x, y)-W_{*}\right)\left(a y-r_{2}\right) \text {. }
$$

According to Theorem 2.21 in Fradkov e Pogromsky (1998, pag. 101) the following SG algorithm

$$
u(t)=-\gamma\left(W(x(t), y(t))-W_{*}\right)\left(a y(t)-r_{2}\right)
$$

achieves the goal (19) for $\gamma>0$ and almost all initial conditions satisfying $x(t)>0, y(t)>0$.

To illustrate the theoretical results we carried out computer simulation of the model (17). The SG algorithm (20) for the system (17) with the following parameter values $r_{1}=1$, $r_{2}=1, a=1, b=1$ is as follows

$$
W(x, y)=(x-1-\log (x))+(y-1-\log (y))
$$

and $u(t)$ is

$$
u(t)=-\gamma\left(W(x(t), y(t))-W_{*}\right)(y(t)-1) .
$$

\section{Simulation of the behavior of the Lotka- Volterra model subject to the control ac- cording to Fradkov et al.}

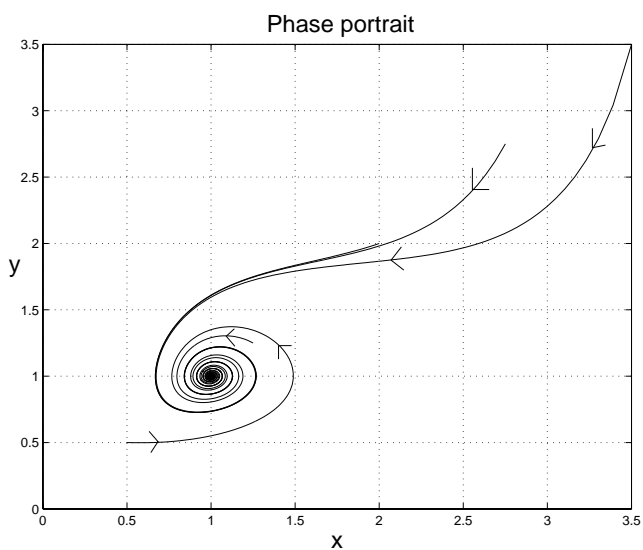

(a)

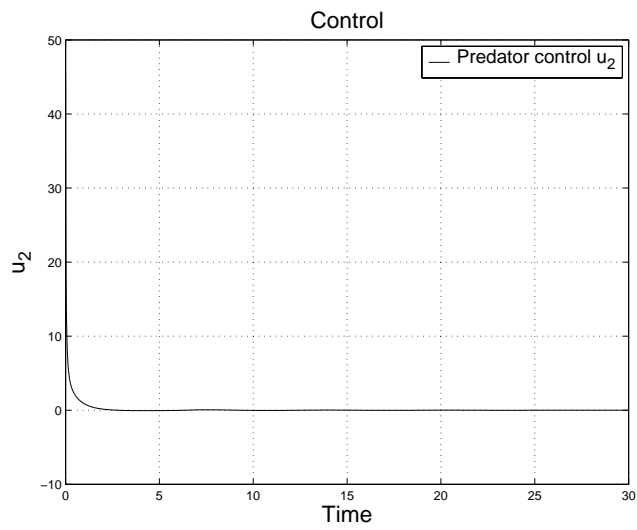

(b)

Figure 4: (a) Phase plane of the Lotka-Volterra model subject to a SG algorithm. (b) Time evolution of the controlling action $u$. Parameter values $r_{1}=1, r_{2}=1, a=1, b=1, \gamma=2$ and $W_{*}=-0.1$.

It is seen that choosing different values of the desired "energy" level $W_{*}$ we can achieve significantly different behavior of the controlled system, as shown in Figures 4 and 5 (Fradkov e Pogromsky, 1998). In the case where $W_{*}=$ -0.1 , the system approaches asymptotically to the equilibrium point $\left(r_{1} / a, r_{2} / b\right)$ as can be observed in Figure 4 and in the case where $W_{*}=0.5$ the system displays a limit cycle as can be observed in Figure 5.

\subsection{Control of systems in the presence of uncertain inputs}

Consider the Lotka-Volterra model under the effect of a harvesting strategy with constant efforts in both species, $h_{1} x$ and $h_{2} y$, and perturbations denoted as $s_{1}(t)$ and $s_{2}(t)$ are 


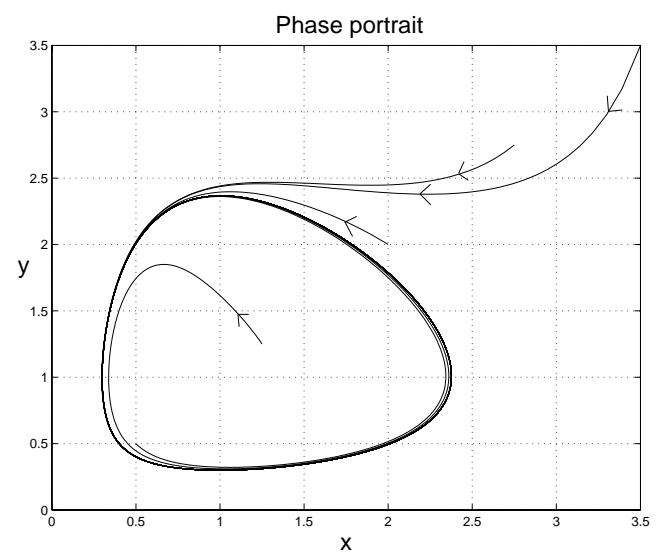

(a)

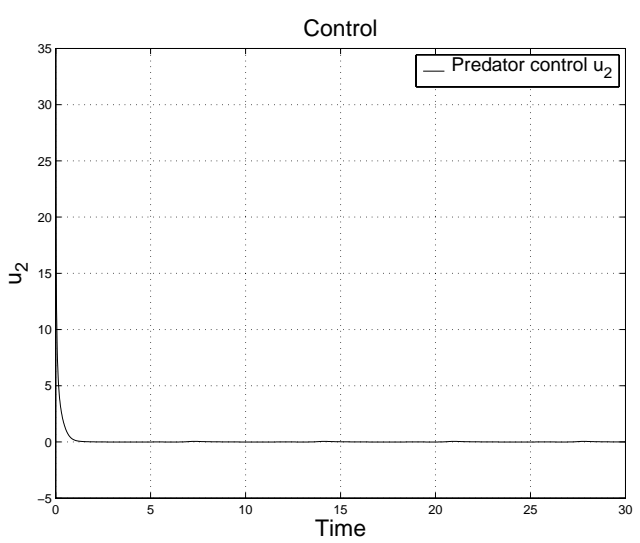

(b)

Figure 5: (a) Phase plane of the Lotka-Volterra model subject to a SG algorithm. (b) Time evolution of the controlling action $u$. Parameter values $r_{1}=1, r_{2}=1, a=1, b=1, \gamma=2$ and $W_{*}=0.5$.

added, as well as additional controls $p_{1}$ and $p_{2}$, as follows:

$$
\begin{aligned}
& \dot{x}=r_{1} x-a x y-x s_{1}+p_{1}-h_{1} x, \\
& \dot{y}=-r_{2} y+b x y-y s_{2}+p_{2}-h_{2} y .
\end{aligned}
$$

The uncertainty is such that $\left|s_{1}\right| \leq s_{m_{1}}$ and $\left|s_{2}\right| \leq s_{m_{2}}$. The corresponding equilibrium point is $\left(x^{*}, y^{*}\right)$. The problem is to maintain this equilibrium point under the uncertainties $s_{1}$ and $s_{2}$ using the controls $p_{1}$ and $p_{2}$.

According to the method in Vincent et al. (1985), the idea is to use knowledge of the reachable set $R$ to calculate the extreme effects of the uncertainty over this set and then use this information in feedback controller design.

A Liapunov function for (21) with $s_{1}=s_{2}=p_{1}=p_{2}=0$, also based on the first integral, is given as follows

$$
V(x, y)=x-x^{*}-x^{*} \ln \left(\frac{x}{x^{*}}\right)+y-y^{*}-y^{*} \ln \left(\frac{y}{y^{*}}\right)
$$

which is valid throughout the region $X$ defined by

$$
X=\left\{x \in \mathbb{R}^{2} \mid x>0, \quad y>0\right\} .
$$

The region $R$ depends on the specific parameters used and the equilibrium points of (21) with $p_{1}=p_{2}=0, s_{1}= \pm s_{m_{1}}$, $s_{2}= \pm s_{m_{2}}$ and $s_{m_{1}}=0.2, s_{m_{2}}=0.15, h_{1}=0.25, h_{2}=$ 0.25 . Therefore, $x^{*}=1.25, y^{*}=0.75, \rho_{1}=1.45 \times 0.2$, $\rho_{2}=0.95 \times 0.15$ and $d=0.25$, we obtain $s_{m_{1}}=0.2$, $s_{m_{2}}=0.15, h_{1}=0.25, h_{2}=0.25$. Therefore, $x^{*}=1.25$, $y^{*}=0.75, \rho_{1}=1.45 \times 0.2, \rho_{2}=0.95 \times 0.15$ and $d=0.25$, we obtain

$$
\left.\begin{array}{l}
\frac{\partial V}{\partial x}=1-\frac{x^{*}}{x} \triangleq e_{1} \\
\frac{\partial V}{\partial y}=1-\frac{y^{*}}{y} \triangleq e_{2}
\end{array}\right\}
$$

Let $\omega=\sqrt{\left(x-x^{*}\right)^{2}+\left(y-y^{*}\right)^{2}}$, then the control laws become

$$
\begin{aligned}
& p_{1}=\left\{\begin{array}{llc}
-\rho_{1} \operatorname{sign}\left(e_{1}\right) & \text { if } & \left|e_{1}\right|>\zeta \\
-\rho_{1} e_{1} / \zeta & \text { if } & \left|e_{1}\right| \leq \zeta \\
-\rho_{1} \exp \left[-l_{1}(\omega-d)\right] \operatorname{sign}\left(e_{1}\right) & \text { if } & \omega>d
\end{array}\right. \\
& p_{2}=\left\{\begin{array}{llc}
-\rho_{2} \operatorname{sign}\left(e_{2}\right) & \text { if } & \left|e_{2}\right|>\zeta \\
-\rho_{2} e_{2} / \zeta & \text { if } & \left|e_{2}\right| \leq \zeta \\
-\rho_{2} \exp \left[-l_{2}(\omega-d)\right] \operatorname{sign}\left(e_{2}\right) & \text { if } & \omega>d
\end{array}\right.
\end{aligned}
$$

\section{Simulation of the behavior of the Lotka- Volterra model subject to the control ac- cording to Vincent et al.}

Consider the following parameter values: $r_{1}=r_{2}=a=$ $b=1, x^{*}=1.25, y^{*}=0.75, \zeta=0.01, l_{1}=1, l_{2}=1$, $s_{1}(t)=0.2 \cos (t), s_{2}(t)=0.15 \cos (t)$.

Figure 6.a shows the simulation of model (21) under perturbations of type $s_{1}(t)=-0.20 \cos (t), s_{2}(t)=-0.15 \cos (t)$ and subject to the control of type (25), (26). Figure 6.b shows the time evolution of the control action.

\subsection{Static sliding-mode control}

Consider a nonlinear unstable plant

$$
\dot{\mathbf{z}}=\mathbf{a}(\mathbf{z})+\mathbf{B}(\mathbf{z}) \xi, \quad \mathbf{z}(0) \neq \mathbf{0} .
$$

The goal is to define a control $\xi$ such that $\mathbf{z}(t) \rightarrow \mathbf{0}$ for $t \rightarrow$ $\infty$. This is achieved by defining a suitable switching surface

$$
\sigma=\mathbf{r}(\mathbf{z})-\mathbf{D}(\mathbf{z}) \operatorname{sign}(\sigma)
$$

where $\mathbf{r}(\mathbf{z}), \mathbf{D}(\mathbf{z})$ must be chosen. The following theorem yields sufficient conditions for the existence of such a staticmode stabilizing control. 


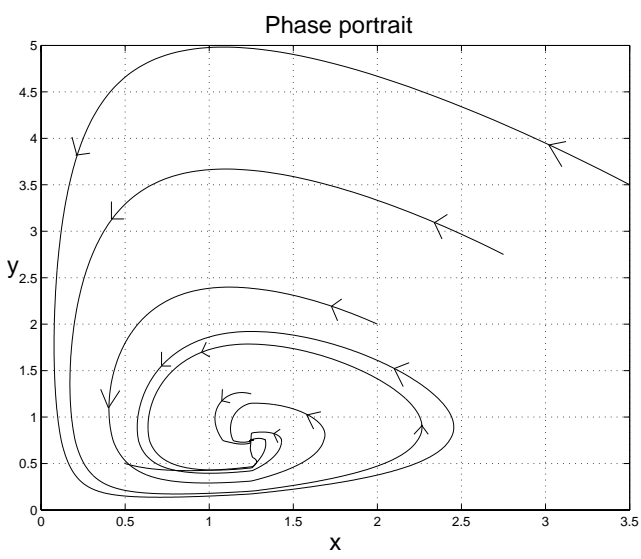

(a)

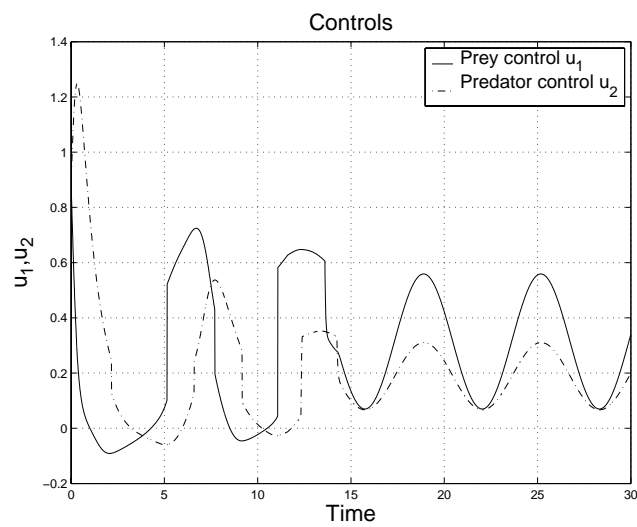

(b)

Figure 6: (a) Phase plane of the Lotka-Volterra model subject to the control according to Vincent. (b) Time evolution of the controlling actions $u_{1}$, $u_{2}$

Junger e Steil (2003) show how the static sliding-mode approach can be effectively applied for nonlinear plant control. They show that the functions $\mathbf{r}(\mathbf{z})$ and $\mathbf{D}(\mathbf{z})$, which were assumed to be given previously, can effectively be constructed for an interesting and large class of nonlinear systems. They defined the sliding-mode control as follows.

Definition 3.1 (Sliding-Mode Control. Definition VI.1 in Junger e Steil (2003)): If $\xi(t)$ guarantees $\sigma(t) \equiv \mathbf{0}, \forall t \in$ $\left[t_{1}, t_{2}\right]$, then it is called sliding-mode control.

Theorem 2 (Theorem VI.1 in Junger e Steil (2003)) Let $\mathbf{v}(\mathbf{z})$ be an $r$-dimensional continuous vector-function such that $\mathbf{z}(t) \rightarrow 0$, whenever $\mathbf{v}(\mathbf{z}) \rightarrow 0$. Assume that $\operatorname{det}\left[\mathbf{J}_{v}(\mathbf{z}) \mathbf{B}(\mathbf{z})\right] \neq 0$ for all $\mathbf{z} \neq 0$, where $\mathbf{J}_{v}(\mathbf{z})$ is the Jacobian matrix of $\mathbf{v}(\mathbf{z})$, then there exists a stabilizing static sliding-mode control.

\section{Constructive procedure for control design (and proof of Theorem 2)}

With regard to the plant (27) the derivative of $\mathbf{v}(\mathbf{z})$ is

$$
\dot{\mathbf{v}}=\mathbf{J}_{v}(\mathbf{z})[\mathbf{a}(\mathbf{z})+\mathbf{B}(\mathbf{z}) \xi] .
$$

Define the system of differential equations

$$
\dot{\mathbf{v}}=-\mathbf{K} \mathbf{v}
$$

where $\mathbf{K}=\operatorname{diag}\left\{k_{i}\right\}>0$ is an arbitrary $r \times r$ constant positive diagonal matrix.

Substitution from (29) yields

$$
\mathbf{J}_{v}(\mathbf{z})(\mathbf{a}(\mathbf{z})+\mathbf{B}(\mathbf{z}) \xi)=-\mathbf{K} \mathbf{v}(\mathbf{z}) .
$$

The sliding surface is defined by following relation:

$$
\sigma=\mathbf{J}_{v}(\mathbf{z})(\mathbf{a}(\mathbf{z})+\mathbf{B}(\mathbf{z}) \xi)+\mathbf{K} \mathbf{v}(\mathbf{z}) .
$$

Then, $\mathbf{r}(\mathbf{z}, t)=\mathbf{J}_{v}(\mathbf{z}) \mathbf{a}(\mathbf{z})+\mathbf{K v}(\mathbf{z}), \mathbf{D}(\mathbf{z}, t)=$ $-\mathbf{J}_{v}(\mathbf{z}) \mathbf{B}(\mathbf{z})$ and $\sigma \equiv 0$ by construction. Now, the system is in sliding mode whenever the static sliding-mode control

$$
\xi=-\left(\mathbf{J}_{v}(\mathbf{z}) \mathbf{B}(\mathbf{z})\right)^{-1}\left(\mathbf{J}_{v}(\mathbf{z}) \mathbf{a}(\mathbf{z})+\mathbf{K} \mathbf{v}(\mathbf{z})\right)
$$

is applied. Under (33), equality (30) holds. Therefore, $\mathbf{v}(\mathbf{z}) \rightarrow 0$ and, hence $\mathbf{z}(t) \rightarrow 0$.

\section{Junger et al. procedure applied to the Lotka-Volterra model}

Consider a nonlinear system of type Lotka-Volterra that we desire to stabilize with the help of the static sliding-mode approach. Assume that the plant (27) has the following parameters:

$$
\mathbf{a}(\mathbf{z})=\left(\begin{array}{c}
r_{1} x-a x y \\
-r_{2} y+b x y
\end{array}\right), \mathbf{B}(\mathbf{z})=\left(\begin{array}{l}
-x \\
-y
\end{array}\right) .
$$

Remark 3.1 Note that the static sliding-mode control is applied to both species.

Chose the function $\mathbf{v}(\mathbf{z})$ as $\left[\begin{array}{ll}x-x_{t h} & y-y_{t h}\end{array}\right]$. The Jacobian $\mathbf{J}_{v}(\mathbf{z}) \mathbf{B}(\mathbf{z})=\left[\begin{array}{ll}-x & -y\end{array}\right]$ is nonzero for all $z \neq 0$.

The corresponding stabilizing static sliding mode continuous control has the form

$\xi=\frac{r_{1} x-a x y-r_{2} y+b x y+k\left(x-x_{t h}\right)+k\left(y-y_{t h}\right)}{x+y}$.

The Lotka-Volterra system under the static sliding mode control is as follows

$$
\left\{\begin{array}{l}
\dot{x}=r_{1} x-a x y-x \xi \\
\dot{y}=-r_{2} y+b x y-y \xi .
\end{array}\right.
$$


Simulation of the behavior of the LotkaVolterra model subject to the control according to Junger et al.

Figure 7 shows the dynamics of the Lotka-Volterra system subject to the static sliding mode control.

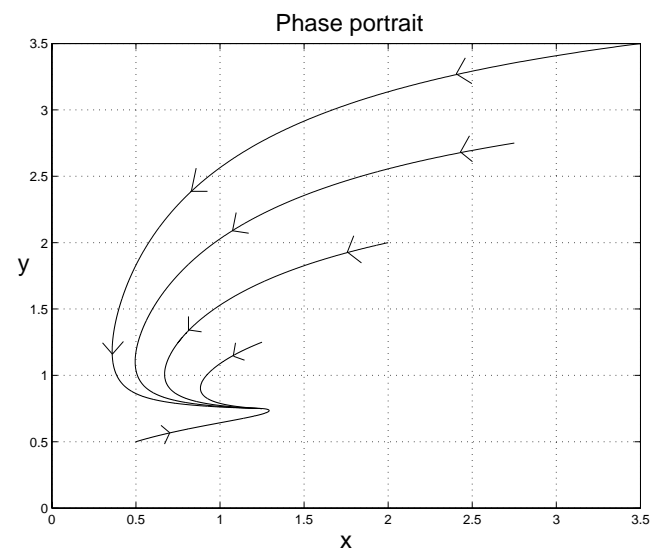

(a)

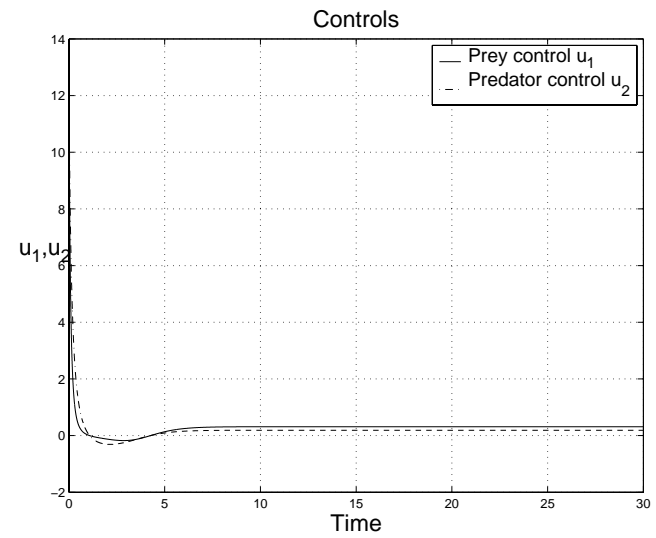

(b)

Figure 7: (a) Phase plane of the Lotka-Volterra model subject to the static sliding mode continuous control. (b) Time evolution of the controlling actions. Parameter values $r_{1}=1, r_{2}=1, a=1, b=1, k=1.25$, $x_{t h}=1.25$ and $y_{t h}=0.75$.

\subsection{Immersion and Invariance for Stabi- lization of Nonlinear Systems}

Consider the Lotka-Volterra system with a control of type Immersion and Invariance (I \& I) applied only to the predator as follows

$$
\left\{\begin{array}{l}
\dot{x}=r_{1} x-a x y \\
\dot{y}=-r_{2} y+b x y+u_{2}
\end{array}\right.
$$

with $z=\left[\begin{array}{ll}x & y\end{array}\right]^{T}, u_{2} \in \mathbb{R}, n=2, p=m=1$ and the following mappings are defined:

$$
\begin{array}{llll}
\alpha(\cdot) \quad: & \mathbb{R} \rightarrow \mathbb{R} \quad \pi(\cdot): \mathbb{R} \rightarrow \mathbb{R}^{2} \quad c(\cdot): \mathbb{R} \rightarrow \mathbb{R} \\
\phi(\cdot) \quad: & \mathbb{R}^{2} \rightarrow \mathbb{R} \quad \psi(\cdot, \cdot): \mathbb{R}^{2 \times 1} \rightarrow \mathbb{R}
\end{array}
$$

such that the following hold.

H1) (Target system) Choose the system

$$
\dot{\xi}=-\xi+x_{t h}
$$

with $\xi \in \mathbb{R}$ and such that it has a globally asymptotically stable equilibrium at $\xi^{*}=x_{t h}$ and

$$
\begin{aligned}
x^{*} & =\pi_{1}\left(\xi^{*}\right)=x_{t h} \\
y^{*} & =\pi_{2}\left(\xi^{*}\right)
\end{aligned}
$$

then

$$
x=\pi_{1}(\xi)=2 \xi-x_{t h}
$$

from the first equation of (37) we obtain

$$
2\left(-\xi+x_{t h}\right)=r_{1}\left(2 \xi-x_{t h}\right)-a\left(2 \xi-x_{t h}\right) \pi_{2}
$$

then

$$
\pi_{2}=\frac{r_{1}\left(2 \xi-x_{t h}\right)+2 \xi-2 x_{t h}}{a\left(2 \xi-x_{t h}\right)} .
$$

H2) (Immersion condition) The function $c(\xi)$ is defined implicitly as:

$$
-r_{2} \pi_{2}+b\left(2 \xi-x_{t h}\right) \pi_{2}+c(\xi)=\frac{\partial \pi_{2}}{\partial \xi}\left(2 \xi-x_{t h}\right) .
$$

H3) (Implicit manifold) The manifold $z=\pi(\xi)$ can be described by

$$
\phi(x, y)=y-\frac{r_{1} x+x-x_{t h}}{a x}
$$

H4) (Manifold attractivity and trajectory boundedness) The dynamics on the manifold is calculated as

$$
\dot{v}=\frac{\partial \phi}{\partial z}[f(z)+g(z) \psi(z, v)]
$$

then

$$
\begin{gathered}
\dot{v}=\left(\begin{array}{cc}
-\frac{x_{t h}}{a x^{2}} & 1
\end{array}\right)\left(\begin{array}{c}
r_{1} x-a x y \\
-r_{2} y+b x y+\psi(z, v)
\end{array}\right) \\
\dot{v}=-\frac{x_{t h}}{a x^{2}}\left(r_{1} x-a x y\right)-r_{2} y+b x y+\psi(z, v) .
\end{gathered}
$$

The design I \& I is completed by choosing

$$
\psi(z, v)=\frac{x_{t h}}{a x^{2}}\left(r_{1} x-a x y\right)+r_{2} y-b x y-v,
$$


which produces the closed loop dynamics

$$
\left\{\begin{aligned}
\dot{x} & =r_{1} x-a x y \\
\dot{y} & =\frac{x_{t h}}{a x^{2}}\left(r_{1} x-a x y\right)-v \\
\dot{v} & =-v .
\end{aligned}\right.
$$

Hence, to complete the design it only remains to show that all trajectories of (39) are bounded. Consider the coordinate transformation

$$
\eta=y-\frac{r_{1} x+x-x_{t h}}{a x}
$$

yielding

$$
\left\{\begin{array}{l}
\dot{x}=-(a \eta+1) x+x_{t h} \\
\dot{\eta}=-v \\
\dot{v}=-v .
\end{array}\right.
$$

Note that $x(t), \eta(t)$ and $v(t)$ are bounded for all $t$ and the control law is obtained as

$$
\begin{aligned}
u_{2}=\psi(z, v)-\eta= & \frac{x_{t h}}{a x^{2}}\left(r_{1} x-a x y\right)+r_{2} y \\
& -b x y-y+\frac{r_{1} x+x-x_{t h}}{a x} .
\end{aligned}
$$

\section{Simulation of the behavior of the Lotka- Volterra model subject to the control ac- cording to Astolfi et al.}

Figure 8 show the dynamics of the Lotka-Volterra system under the control law I \& I.

\section{COMPARISON OF THE DIFFERENT CONTROL TECHNIQUES}

We use the terminology established in section 2 to make a comparison of the different techniques in a tabular form:

Table 1 shows that only the proposed control possesses all the desirable charateristics specified in Section 2. To be completely fair, it should be pointed out that we have not explicitly compared control with respect to robustness, although it is well known (Utkin, 1992) that all variable structure designs, such as the one proposed in this paper, have an inherent robustness to bounded uncertainty. On the other hand, given the considerably greater difficulty, or even impossibility, in the implementation of the other controls, it seems reasonable to limit our comparison to the items in the columns of Table 1.

\section{CONCLUDING REMARKS}

The proposed control possess all the desirable characteristics of a control to be applied in an ecological context, i.e. (i)

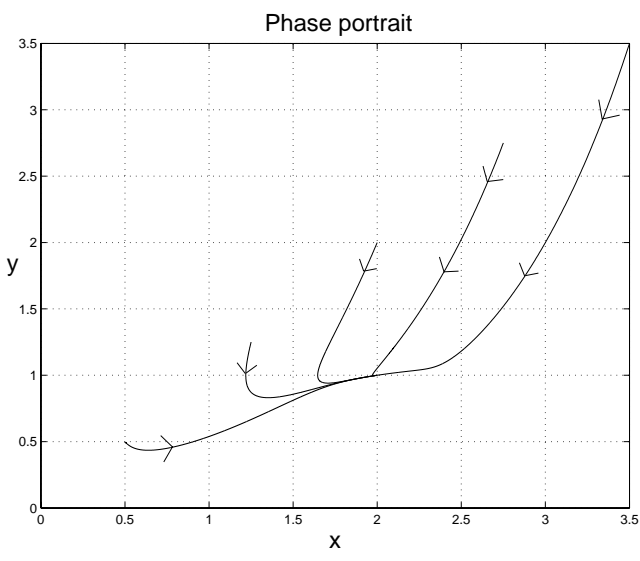

(a)

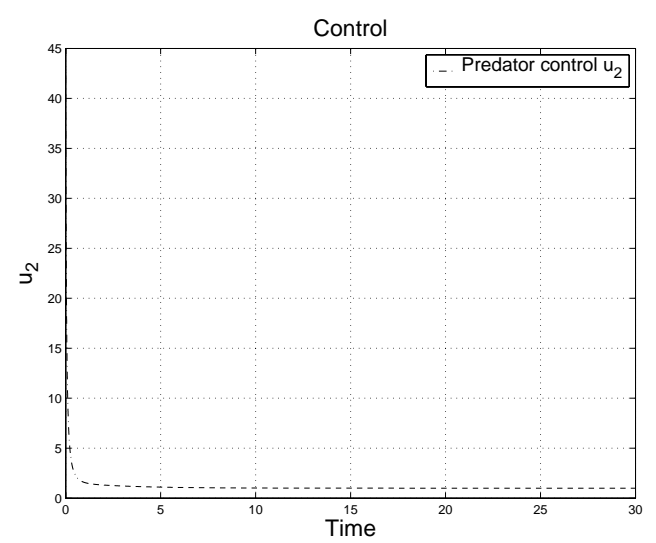

(b)

Figure 8: (a) Phase plane of the Lotka-Volterra model subject to the control I \& I. (b) Time evolution of the controlling action. Parameter values $r_{1}=1, r_{2}=1, a=1, b=1, k=1$ and $x_{t h}=2$.

easy to implement, i.e., it is a proportional control; (ii) the control is carried through the removal of only one species; (iii) only one species needs to be monitored; and (iv) species coexistence is achieved. Moreover, in comparison with several existing methods, both old and new, it seems to be the only one that combines all these desirable characteristics.

In terms of future work along the lines initiated in this paper, we mention a few topics.

In the real world, the growth rate of a particular species is usually not a function of the current population density, but rather that of a density at some point in the recent past. In other words, there is a delay in the functional response. It is also well known (May, 1973; Kuang, 1993) that the inclusion of delays in the system model can have unexpected effects, often, but not always, destabilizing. It is thus necessary to carry out a detailed and rigorous study of system behavior when delays are present, either in the state or in the control. 
Table 1: Comparison of control techniques for the Lotka-Volterra system

\begin{tabular}{|c|c|c|c|}
\hline Control Characteristics & Implementation & Nonnegative & Monitoring \\
\hline Emel'yanov & Difficult & Yes & 2 species \\
\hline Fradkov & Difficult & No & 2 species \\
\hline Vincent & Difficult & No & 2 species \\
\hline Junger & Difficult & No & 2 species \\
\hline I \& I & Difficult & Yes & $\mathbf{1}$ species \\
\hline Proposed & Easy & Yes & \\
\hline
\end{tabular}

Some pointers to technical results that may be useful in this context are Tarbouriech et al. (2000), Mazenc e Niculescu (2001), Dercole et al. (2003).

Models of virus dynamics (Nowak e May, 2000) are very similar to the predator-prey models studied in this paper. There is great current interest in systematically finding "protocols"(controls) that are capable of stabilizing virus populations at low levels (Wein et al., 1997) and, once again, desirable methods must have most of the characteristics stipulated in Section 2. We expect that the control design proposed in this paper will be applicable to this class of problems as well.

Finally, there has been recent interest in applying bifurcation analysis to planar population dynamics models, and preliminary work of this kind can be found in Kuznetsov et al. (2003), Cunha et al. (2003), Moreno et al. (2003).

\section{ACKNOWLEDGMENT}

This research was partially financed by Project Nos. 140811/2002-8, 551863/2002-1, 471262/03-0 of CNPq, No. E-26/150.505/2002, E-26/152.177/2003 of FAPERJ and also by the agency CAPES. Corresponding authors: Magno E. Mendoza Meza, Amit Bhaya.

\section{REFERENCES}

Astolfi, A. e Ortega, R. (2003). Immersion and invariance: A new tool for stabilization and adaptive control of nonlinear systems, IEEE Trans. Automat. Con- trol 46(4): 590-606.

Beddington, J. R. e May, R. M. (1977). Harvesting natural populations in a randomly fluctuating environment, $\mathrm{Sci}$ ence 197: 463-465.

Costa, M. I. S., Kaszkurewicz, E., Bhaya, A. e Hsu, L. (2000). Achieving global convergence to an equilibrium population in predator-prey systems by the use of discontinuous harvesting policy, Ecological Modelling 128: 89-99.

Cunha, F. B., Pagano, D. J. e Moreno, U. F. (2003). Sliding bifurcations of equilibria in planar variable structure systems, IEEE Trans. Circuits and Systems-I: Fundamental Theory and Applications 50(8): 1129-1134.

Dercole, F., Gragnani, A., Kuznetsov, Y. A. e Rinaldi, S. (2003). Numerical sliding bifurcation analysis: An application to a relay control system, IEEE Trans. Circuits and Systems-I: Fundamental Theory and Applications 50(8): 1058-1063.

Emel'yanov, S. V., Burovoi, I. A. e Levada, F. Y. (1998). Control of Indefinite Nonlinear Dynamics Systems, Vol. 231 of Lecture Notes in Control and Information Sciences, Springer - Verlag, Great Britain.

Fradkov, A. L. e Pogromsky, A. Y. (1998). Introduction to Control of Oscillations and Chaos, Vol. 35 of Nonlinear Science, World Scientific, Singapore.

Gurney, W. S. C. e Nisbet, R. M. (1998). Ecological dynamics, Oxford University Press, New York. 
Junger, I. B. e Steil, J. J. (2003). Static sliding-motion phenomena in dynamical systems, IEEE Trans. Automat. Control 48(4): 680-686.

Khalil, H. K. (1992). Nonlinear Systems, Macmillan Publishing.

Kuang, Y. (1993). Delay Differential Equations with Applications in Population Dynamics, Academic Press, San Diego.

Kuznetsov, Y. A., Rinaldi, S. e Gragnani, A. (2003). One-parameter bifurcations in planar Filippov systems, International Journal of Bifurcation and Chaos 13(8): 2157-2188.

Lee, C. S. e Leitmann, G. (1983). On optimal long-term management of some ecological systems subject to uncertain disturbances, Internat. J. Systems Science 14(8): 979-994.

May, R. (1973). Stability and Complexity in Model Ecosystems, Princeton University Press.

Mazenc, F. e Niculescu, S.-I. (2001). Lyapunov stability analysis for nonlinear delay systems, Systems and Control Letters 42(4): 245-251.

Meza, M. E. M. (2004). Nonlinear systems of the predator-prey type: Control design using Liapunov Functions. Available at http://www.nacad.ufrj.br/_amit/teses_dsc_or/ tese_dsc_meza2004.pdf.

Moreno, U. F., Peres, P. L. D. e Bonatti, I. S. (2003). Analysis of piecewise-linear oscillators with hysteresis, IEEE Trans. Circuits and Systems-I: Fundamental Theory and Applications 50(8): 1120-1124.

Nowak, M. A. e May, R. M. (2000). Virus dynamics: Mathematical principles of immunology and virology, Oxford University Press, Oxford.

Sepulchre, R., Janković, M. e Kokotović, P. (1997). Constructive Nonlinear Control, Series on Communications and Control Engineering (CCES), SpringerVerlag, London.

Slotine, J.-J. E. e Li, W. (1991). Applied Nonlinear Control, Prentice Hall, Englewood Cliffs, New Jersey.

Sontag, E. D. (1989). A 'universal' construction of Artstein's theorem on nonlinear stabilization, Systems and Control Letters 13: 117-123.

Steele, J. H. e Henderson, E. W. (1984). Modeling long-term in fish stocks, Science 224: 985-987.
Tarbouriech, S., Peres, P. L. D., Garcia, G. e Queinnec, I. (2000). Delay-dependent stabilization of time-delay systems with saturating actuators, Proceeding of the $39^{\text {th }}$ IEEE Conference on Decision and Control, Sydney, Australia, pp. 3248-3253.

Utkin, V. I. (1992). Sliding Modes In Control And Optimization, Springer-Verlag, Berlin.

Vincent, T. L., Lee, C. S. e Goh, B. S. (1985). Maintenance of an equilibrium state in the presence of uncertain inputs, Internat. J. Systems Science 16(11): 1335-1344.

Wein, L. M., Zenios, S. A. e Nowak, M. A. (1997). Dynamic multidrug therapies for HIV: A control theoretic approach, J. Theor. Biol. 185(1): 15-29. 